\title{
Towards resolution of bridging descriptions
}

\author{
Renata Vieira and Simone Teufel \\ Centre for Cognitive Science - University of Edinburgh \\ 2, Buccleuch Place EH8 9LW Edinburgh UK \\ \{renata, simone\}@cogsci.ed.ac.uk
}

\begin{abstract}
We present preliminary results concerning robust techniques for resolving bridging definite descriptions. We report our analysis of a collection of 20 Wall Street Journal articles from the Penn Treebank Corpus and our experiments with WordNet to identify relations between bridging descriptions and their antecedents.
\end{abstract}

\section{Background}

As part of our research on definite description (DD) interpretation, we asked 3 subjects to classify the uses of DDs in a corpus using a taxonomy related to the proposals of (Hawkins, 1978) (Prince, 1981) and (Prince, 1992). Of the 1040 DDs in our corpus, $312(30 \%)$ were identified as anaphoric (same head), $492(47 \%)$ as larger situation/unfamiliar (Prince's discourse new), and $204(20 \%)$ as bridging references, defined as uses of DDs whose antecedentscoreferential or not-have a different head noun; the remaining were classified as idioms or were cases for which the subjects expressed doubt - see (Poesio and Vieira, 1997) for a description of the experiments.

In previous work we implemented a system capable of interpreting DDs in a parsed corpus (Vieira and Poesio, 1997). Our implementation employed fairly simple techniques; we concentrated on anaphoric (same head) descriptions (resolved by matching the head nouns of DDs with those of their antecedents) and larger situation/unfamiliar descriptions (identified by certain syntactic structures, as suggested in (Hawkins, 1978)). In this paper we describe our subsequent work on bridging DDs, which involve more complex forms of commonsense reasoning.

\section{Bridging descriptions: a corpus study}

Linguistic and computational theories of bridging references acknowledge two main problems in their resolution: first, to find their antecedents (ANCHORS) and second, to find the relations (LINKS) holding between the descriptions and their anchors (Clark, 1977; Sidner, 1979; Heim, 1982; Carter, 1987; Fraurud, 1990; Chinchor and Sundheim, 1995; Strand, 1997). A speaker is licensed in using a bridging $\mathrm{DD}$ when he/she can assume that the commonsense knowledge required to identify the relation is shared by the listener (Hawkins, 1978; Clark and Marshall, 1981; Prince, 1981). This reliance on shared knowledge means that, in general, a system could only resolve bridging references when supplied with an adequate lexicon; the best results have been obtained by restricting the domain and feeding the system with specific knowledge (Carter, 1987). We used the publicly available lexical database WordNet (WN) (Miller, 1993) as an approximation of a knowledge basis containing generic information.

Bridging DDs and WordNet As a first experiment, we used WN to automatically find the anchor of a bridging DD, among the NPs contained in the previous five sentences. The system reports a semantic link between the DD and the NP if one of the following is true:

- The NP and the DD are synonyms of each other, as in the suit - the lawsuit.

- The NP and the DD are in direct hyponymy relation with each other, for instance, dollar - the currency.

- There is a direct or indirect meronymy (partof relation) between the NP and the DD. Indirect meronymy holds when a concept inherits parts from its hypernyms, like car inherits the part wheel from its hypernym wheeled_vehicle.

- Due to WN's idiosyncratic encoding, it is often 
necessary to look for a semantic relation between sisters, i.e. hyponyms of the same hypernym, such as home - the house.

An automatic search for a semantic relation in 5481 possible anchor/DD pairs (relative to 204 bridging DDs) found a total of 240 relations, distributed over 107 cases of DDs. There were 54 correct resolutions (distributed over 34 DDs) and 186 false positives.

Types of bridging definite descriptions A closer analysis revealed one reason for the poor results: anchors and descriptions are often linked by other means than direct lexico-semantic relations. According to different anchor/link types and their processing requirements, we observed six major classes of bridging DDs in our corpus:

Synonymy/Hyponymy/Meronymy These DDs are in a semantic relation with their anchors that might be encoded in WN. Examples are: a) Synonymy: new album - the record, three bills the legislation; b) Hypernymy-Hyponymy: rice the plant, the television show - the program; c) Meronymy: plants - the pollen, the house - the chimney.

Names Definite descriptions may be anchored to proper names, as in: Mrs. Park - the housewife and Pinkerton's Inc - the company.

Events There are cases where the anchor of a bridging DD is not an NP but a VP or a sentence. Examples are: ...individual investors contend. - They make the argument in letters...; Kadane Oil Co. is currently drilling two wells... - The activity ...

Compound Nouns This class of DDs requires considering not only the head nouns of a DD and its anchor for its resolution but also the premodifiers. Examples include: stock market crash - the markets, and discount packages - the discounts.

Discourse Topic There are some cases of DDs which are anchored to an implicit discourse topic rather than to some specific NP or VP. For instance, the industry (the topic being oil companies) and the first half (the topic being a concert).

Inference One other class of bridging DDs includes cases based on a relation of reason, cause, consequence, or set-members between an anchor (previous NP) and the DD (as in Republicans/Democratics the two sides, and last week's earthquake - the suffering people are going through).

The relative importance of these classes in our corpus is shown in Table 1 . These results explain in part the poor results obtained in our first experiment: only $19 \%$ of the cases of bridging DDs fall into the category which we might expect WN to handle.

\begin{tabular}{|l|l|l|l|l|l|}
\hline Class & $\#$ & $\%$ & Class & $\#$ & $\%$ \\
\hline S/H/M & 38 & $19 \%$ & C.Nouns & 25 & $12 \%$ \\
\hline Names & 49 & $24 \%$ & D.Topic & 15 & $07 \%$ \\
\hline Events & 40 & $20 \%$ & Inference & 37 & $18 \%$ \\
\hline
\end{tabular}

Table 1: Distribution of types of bridging DDs

\section{Other experiments with WordNet}

Cases that WN could handle Next, we considered only the 38 cases of syn/hyp/mer relations and tested whether WN encoded a semantic relation between them and their (manually identified) anchors. The results for these 38 DDs are summarized in Table 2 . Overall recall was $39 \%(15 / 38){ }^{1}$

\begin{tabular}{|l|l|l|l|}
\hline Class & Total & Found in WN & Not Found \\
\hline Syn & 12 & 4 & 8 \\
\hline Hyp & 14 & 8 & 6 \\
\hline Mer & 12 & 3 & 9 \\
\hline
\end{tabular}

Table 2: Search for semantic relations in WN

Problems with WordNet Some of the missing relations are due to the unexpected way in which knowledge is organized in WN. For example, our

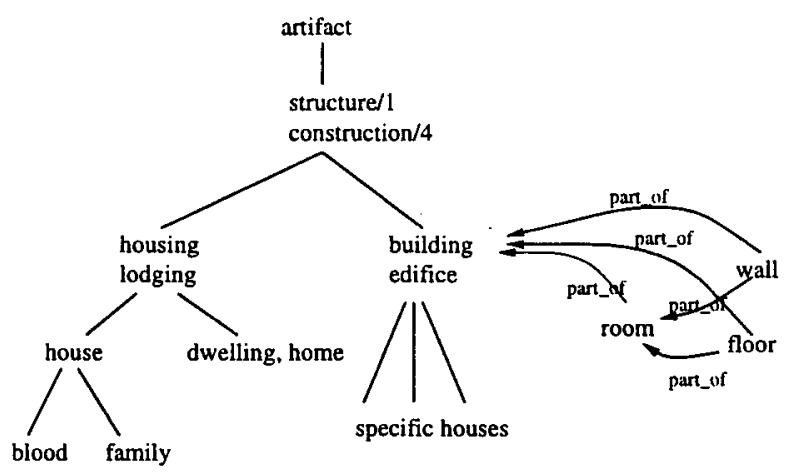

Figure 1: Part of WN's semantic net for buildings

method could not find an association between house and walls, because house was not entered as a hyponym of building but of housing, and housing does

\footnotetext{
${ }^{1}$ Our previous experiment found correct relations for 34 DDs, from which only 18 were in the syn/hyp/mer class. Among these 18, 8 were based on different anchors from the ones we identified manually (for instance, we identified pound - the currency, whereas our automatic search found sterling - the currency). Other 16 correct relations resulting from the automatic search were found for DDs which we have ascribed manually to other classes than syn/hyp/mer, for instance, a relation was found for the pair Bach - the composer, in which the anchor is a name. Also, whereas we identified the pair Koreans - the population, the search found a WN relation for nation - the population.
} 
not have a meronymy link to wall whereas building does. On the other hand, specific houses (schoolhouse, smoke house, tavern) were encoded in WN as hyponyms of building rather than hyponyms of house (Fig. 1).

Discourse structure Another problem found in our first test with $\mathrm{W:N}$ was the large number of false positives. Ideally, we should have a mechanism for focus tracking to reduce the number of false positives - (Sidner. 1979), (Grosz, 1977). We repeated our first experiment using a simpler heuristic: considering only the closest anchor found in a five sentence window (instead of all possible anchors). By adopting this heuristic we found the correct anchors for $30 \mathrm{DDs}$ (instead of 34 ) and reduced the number of false positives from 186 to $i 7$.

\section{Future work}

We are currently working on a revised version of the system that takes the problems just discussed into account. A few names are available in $W N$, such as famous people, countries, cities and languages. For other names, if we can infer their entity type we could resolve them using WN. Entity types can be identified by complements like $\mathrm{Vr}$., Co., Inc. etc. An initial implementation of this idea resulted in the resolution of $53 \%(26 / 49)$ of the cases based on names. Some relations are not found in WN, for instance, $M r$. Morishita (type person) - the 57 year-old. To process DDs based on events we could try first to transform verbs into their nominalisations, and then looking for a relation between nouns in a semantic net. Some rule based heuristics or a stochastic method are required to 'guess' the form of a nominalisation. We propose to use WN's morphology component as a stemmer, and to augment the verbal stems with the most common suffixes for nominalisations, like - ment, -ion. In our corpus, $16 \%$ $(\tau / 43)$ of the cases based on events are direct nominalisations (for instance, changes were proposed the proposals), and another $16 \%$ were based on semantic relations holding between nouns and verbs (such as borrowed - the loan). The other 29 cases $(68 \%)$ of DDs based on events require inference reasoning based on the compositional meaning of the phrases (as in It went looking for a partner - the prospect); these cases are out of reach just now, as well as the cases listed under "discourse topic" and "inference". We still have to look in more detail at compound nouns.

\section{References}

Carter, D. M. 1987. Interpreting Anaphors in .Vatural Language Texts. Ellis Horwood, Chichester. UK.

Chinchor. N. A. and B. Sundheim. 1995. (MUC) tests of discourse processing. In Proc. A.A. I SS on Empirical . Wethods in Discourse Interpretation and Generation. pages 21-26. Stanford.

Clark, H. H. 1977. Bridging. In Johnson-Laird and Wason, eds.. Thinking: Readings in Cognitive Science. Cambridge University Press, Cambridge.

Clark, H. H. and C. R. Marshall. 1981. Definite reference and mutual knowledge. In Joshi, Webber and Sag, eds., Elements of Discourse Understanding. Cambridge University Press, Cambridge.

Fraurud, K. 1990. Definiteness and the Processing of Noun Phrases in Vatural Discourse. Journal of Semantics, $T$, pages $39.5-433$.

Grosz, B. J. 1977. The Representation and lise of Focus in Dialogue Understanding. Ph.D. thesis, Stanford University.

Hawkins, J. A. 1978. Definiteness and Indefiniteness. Croom Helm, London.

Heim, I. 1982. The Semantics of Definite and Indefinite Voun Phrases. Ph.D. thesis, University of Massachuset ts at Amherst.

Miller, G. et al. 1993. Five papers in WordNet. Technical Report CSL Report 43, Cognitive Science Laboratory, Princeton University.

Poesio, M. and Vieira. R. 1997. A Corpus based investigation of definite description use. Manuscript, Centre for Cognitive Science, University of Edinburgh.

Prince, E. 1981. Toward a taxonomy of given/new information. In Cole, ed., Radical Pragmatics. Academic Press. New York, pages 223-255.

Prince, E. 1992. The ZPG letter: subjects, defineteness, and information-status. In Thompson and Mann, eds., Discourse description: diverse analyses of a fund raising tert. Benjamins. Amsterdam, pages 295-325.

Sidner, C. L. 1979. Touards a computational theory of definite anaphora comprehension in English discourse. Ph.D. thesis. WIT.

Strand, K. 1997. A Taxonomy of Linking Relations. Journal of Semantics, forthcoming.

Vieira, R. and M. Poesio. 1997. Corpus-based processing of definite descriptions. In Botley and McEnery eds., Corpus-based and computational approaches to anaphora. UCL Press. London. 